\title{
REVIEW
}

\section{Ovarian inguinal hernia}

\section{A Prodromidou ${ }^{1}$, N Machairas ${ }^{2}$, Z Garoufalia $^{3}$, ID Kostakis ${ }^{3}$, AV Kyriakidis ${ }^{4}$, E Spartalis ${ }^{3}$, GC Sotiropoulos ${ }^{3}$}

${ }^{1}$ Metaxa Memorial Cancer Hospital, Piraeus, Greece

${ }^{2}$ Attikon University Hospital, Athens, Greece

${ }^{3}$ Laiko General Hospital, Athens, Greece

${ }^{4}$ Amfissa General Hospital, Amfissa, Greece

\section{ABSTRACT}

INTRODUCTION Gynaecological structures such as the ovaries, fallopian tubes, ligaments and uterus are rarely encountered inside a hernial sac. The prevalence of groin hernias containing parts of female genitalia remains unknown. The aim of this review was to summarise the existing evidence on inguinal hernias containing ovaries with or without the other female adnexa. METHODS A systematic search was conducted for literature published up to February 2018 using the MEDLINE ${ }^{\circledR}$, Scopus $^{\circledR}$ and Google Scholar ${ }^{\mathrm{TM}}$ databases along with the references of the full-text articles retrieved. Papers on observational studies and case reports concerning women who were diagnosed with an ovarian inguinal hernia (pre or intraoperatively) were considered eligible for inclusion in the review.

RESULTS Fifteen papers (13 case reports, 2 case series) comprising seventeen patients (mean age 47.9 years) were evaluated. A left-sided hernia was noted in 13 cases (77\%) whereas 4 patients had a right-sided hernia. Eight patients underwent preoperative imaging with computed tomography, ultrasonography or both. This was diagnostic in five cases. In 11 patients, hernia contents were repositioned, 2 had a salpingo-oophorectomy and 2 an oophorectomy. Eight patients underwent hernia repair with mesh placement while three had a herniorrhaphy.

CONCLUSIONS Ovarian inguinal hernias should be considered among the differential diagnoses of a groin mass or swelling. In women of reproductive age, repair of the hernia with the intent to preserve fertility is of critical importance.

\section{KEYWORDS}

Ovarian hernia - Inguinal hernia - Ovary - Fertility

Accepted 21 April 2019

CORRESPONDENCE TO

loannis Kostakis, E: i.d.kostakis@gmail.com

\section{Introduction}

Inguinal hernia is defined as the protrusion of an abdominal organ or preperitoneal adipose tissue through the inguinal canal. These hernias represent a frequent benign surgical condition and account for $75 \%$ of all abdominal wall hernias. ${ }^{1}$ The lifetime risk of developing an inguinal hernia ranges from $27 \%$ to $43 \%$ for men and from $3 \%$ to $6 \%$ for women. ${ }^{2}$ They can be detected either through clinical examination for investigation of a groin mass and pain in the inguinal area or when complications arise such as strangulation of the contents of the hernial sac. $^{3}$ Surgical repair constitutes the optimal treatment strategy, especially in symptomatic and complicated hernias. For small hernias and those that are asymptomatic or minimally symptomatic, however, observation has been safely adopted as a management strategy by many clinicians even though surgery for hernia repair is acknowledged as a safe and efficient procedure. ${ }^{4}$

Various organs have been reported to have herniated through the inguinal canal including the small bowel, colon, bladder and (more rarely) the appendix or female adnexa..$^{5}$ There is a paucity of data regarding the prevalence of groin hernias containing parts of female genitalia. According to a retrospective study by Gurer et al, only $7(2.9 \%)$ of the 1,950 patients who underwent hernia repair between 1989 and 2004 had hernias containing ovary and fallopian tube. ${ }^{6}$ The majority of such hernias occur in paediatric cases and they are mostly associated with congenital defects. ${ }^{7}$ In women of reproductive age, taking into account the rarity of this clinical condition, a high clinical suspicion is paramount to ensuring fertility and diagnosing potential simultaneous genital anomalies.

The objective of this systematic review was to summarise the existing evidence on inguinal hernias containing ovaries with or without the other female adnexa. Special consideration was given to their clinical presentation and the diagnostic approach.

\section{Methods}

All appropriate observational studies and case reports concerning women diagnosed with an ovarian inguinal 
hernia (pre or intraoperatively) were considered eligible for inclusion in this systematic review. Reviews and animal studies were excluded. No language restrictions were employed. Two of the authors (NM and AP) independently performed a meticulous search of the literature, excluded duplicate records and compiled the relevant data.

A systematic search was undertaken for literature published up to February 2018 using the MEDLINE ${ }^{\circledR}$ (1966-2018), Scopus ${ }^{\circledR}$ (2004-2018) and Google Scholar ${ }^{\mathrm{TM}}$ (2004-2018) databases along with the references of the full-text articles retrieved. The search keywords were kept to a minimum in an attempt to identify potentially relevant papers that could be easily searched while simultaneously reducing potential loss of eligible articles. The keywords used for the search comprised: 'inguinal hernia', 'ovarian', 'ovarian hernia' and 'inguinal ovary'. Articles deemed to fulfil the inclusion criteria were retrieved. All papers that described cases of women aged $>18$ years who had no known developmental genital tract defect and were found with inguinal hernias containing ovaries (with or without uterus and fallopian tubes) were included in the review.

Data recorded on patient characteristics included age and parity of the women. Characteristics of the hernia (site, reducibility, incarceration) along with clinical appearance and diagnostic methods (primary symptom, clinical examination, imaging) were appraised. Intra and postoperative outcomes were also evaluated. These encompassed hernia contents, type of surgical procedure and hernia repair, length of hospital stay, complications and histopathological outcomes of the excised specimens (if available).

\section{Results}

Figure 1 illustrates the stages of article selection. Twentyseven full-text articles were retrieved for detailed evaluation. Twelve of these were subsequently excluded from the review. Five of them reported ovarian inguinal hernias associated with defects of genital tract development. ${ }^{8-12}$ Three others described hernias containing ovarian cysts. ${ }^{13-15}$ In two case reports, the hernia contents were not of ovarian origin. ${ }^{16,17}$ In another paper, the hernial sac contained a mass that histopathologically mimicked a uterus with a leiomyomatous lesion. ${ }^{18}$ Finally, one article was excluded because the full text could not be found. ${ }^{19}$

Consequently, 15 papers (13 case reports, 2 case series), comprising 17 patients with ovarian inguinal hernias, were included in our review. ${ }^{5,6,20-32}$ Our findings are

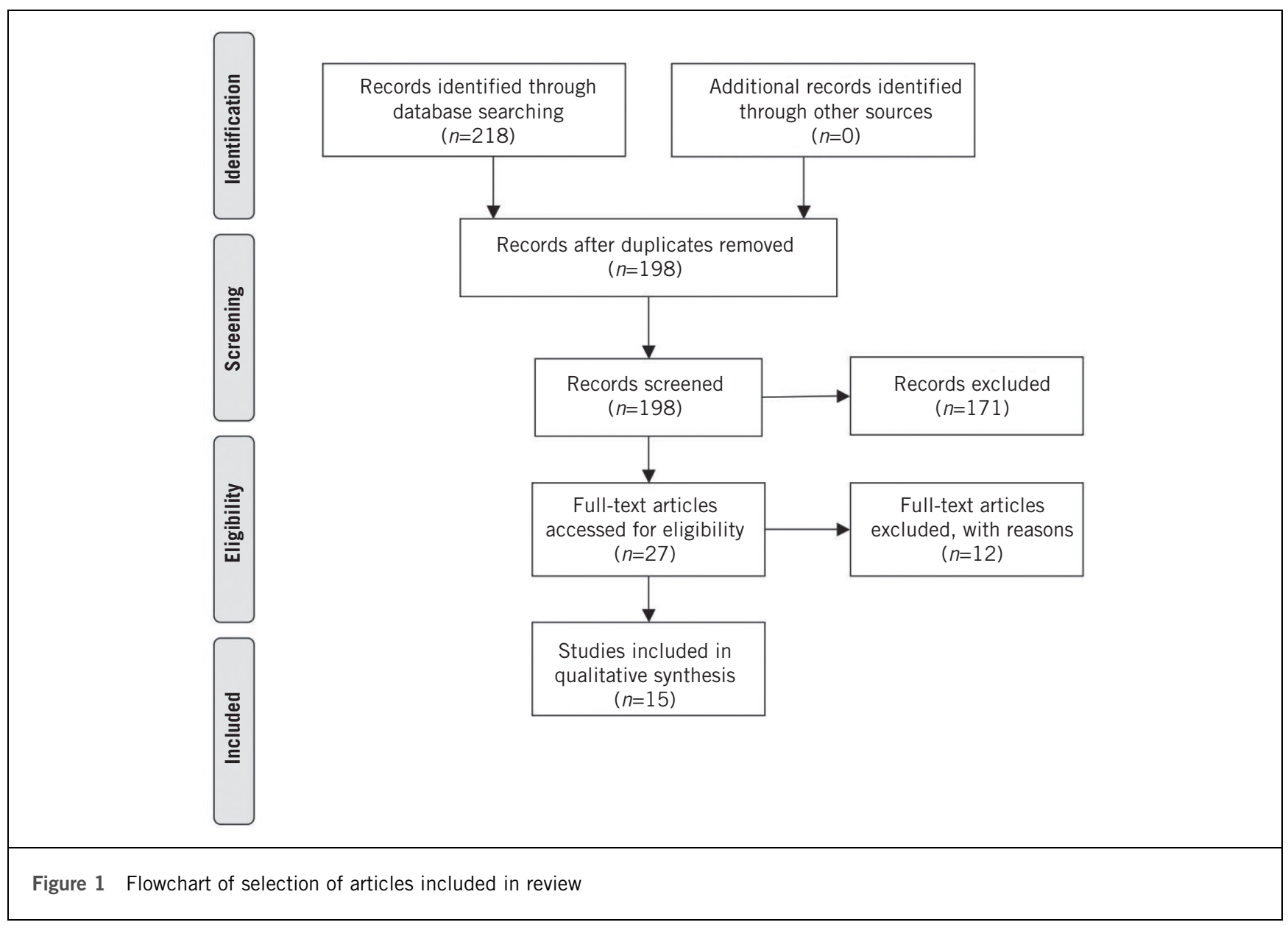


summarised in Tables 1-3. The mean patient age was 47.9 years (standard deviation: 23.7 years) and over half of the patients $(n=9,56 \%)$ were women of reproductive age (18-45 years). Three of the women were nulliparous, with two of these presenting with infertility. Eight had one or more children. For the remaining six cases, data concerning parity were not available.

A left-sided hernia was noted in over three-quarters of the cases $(n=13,77 \%)$. The majority of patients $(n=15$, $88 \%$ ) were admitted with a groin mass or swelling (painful in 7 cases, painless in 8 cases). In the remaining two patients, an ovarian inguinal hernia was identified while investigating abnormal liver function and infertility respectively, and no pain or groin mass were reported.

Imaging was performed in eight cases: three patients underwent preoperative computed tomography (CT), three patients had ultrasonography, and in two patients, both ultrasonography and CT were performed. In five cases, the imaging results indicated herniated gynaecological structures and in two patients, ultrasonography revealed cystic structures inside the hernial sac. Finally, in one woman, ultrasonography showed absence of uterus and ovary at their normal anatomical site.

The diagnosis of a hernia was based solely on clinical examination in five patients; the contents of these hernias could therefore not have been determined preoperatively. A palpable, tender lump was noted in the affected inguinal region in all except 2 of the 17 cases. Fifteen of the hernias were irreducible (with 3 of these also being strangulated), one was reducible and one was non-palpable.

Sixteen of the seventeen patients underwent surgery. On surgical exploration, ovary was the only finding inside the hernia in four cases. Ovary with fallopian tube were identified in eight cases, two hernias contained ovary with fallopian tube and part of the uterus, and in two cases, an ovarian or paraovarian cyst was found along with the ovary and fallopian tube. In 14 cases, the surgical approach was open while 2 patients underwent exploratory laparoscopy. The hernia contents (ovary with or without uterus or fallopian tube) were repositioned in $11(69 \%)$ of the patients undergoing surgery after ensuring organ viability. In two of these cases, in which an ovarian cyst was detected, a cystectomy was performed. Two patients underwent a salpingo-oophorectomy and two underwent an oophorectomy. In one case, no surgical repair was performed.

Information on the type of hernia repair performed was available in 11 of the 16 patients who had surgery. A herniorrhaphy was carried out in three cases (27\%) while eight patients $(73 \%)$ underwent hernia repair with mesh. Details of the hernia repair were not available in four cases and hernia repair was not performed in one case owing to lack of consent.

No deaths or postoperative complications were documented. Information on hospital stay was reported by four authors, with a range of 2-7 days (mean: 4.5 days). Data concerning follow-up were recorded by Ueda et al, Malik et al and Ray et al, who detected no hernia recurrence in the operated patients at nine months, three months and six weeks following surgery respectively. ${ }^{27,28,30}$

\section{Discussion}

Inguinal hernia is a benign condition commonly encountered in the general population. Surgical repair of inguinal hernia accounts for a third of the most frequently

\section{Table 1 Summary of cases included in review}

$\begin{array}{ll}\text { Total number of patients } & 17 \\ \text { Mean age in years } & 47.9 \text { (SD: 23.7) } \\ \text { Hernia side } & 13 \\ \text { Left } & 4 \\ \text { Right } & \\ \text { Symptoms } & 7 \\ \text { Pain } & 15 \\ \text { Groin mass or swelling } & 2 \\ \text { Other symptoms } & \\ \text { Hernia reduction } & 1 \\ \text { Reducible } & 15 \\ \text { Irreducible } & 1 \\ \text { Non-palpable } & \\ \text { Diagnosis of gynaecological structures inside hernia } \\ \text { Preoperative (CT, ultrasonography or both) } & 5 \\ \text { Intraoperative } & 12 \\ \text { Surgical approach } & 14 \\ \text { Open } & 2 \\ \text { Laparoscopic } & 1 \\ \text { No surgery } & \\ \text { Suca } & \end{array}$

Surgical procedure $(n=16)$

Repositioning of structures $\quad 11$

Oophorectomy

2

Salpingo-oophorectomy

Not mentioned

1

Intraoperative findings $(n=16)$

Ovary 4

Ovary + fallopian tube $\quad 8$

Uterus + ovary + fallopian tube 2

Other

2

Mesh placement $(n=11)$

Yes 8

No (herniorrhaphy)

$\mathrm{CT}=$ computed tomography; $\mathrm{SD}=$ standard deviation 


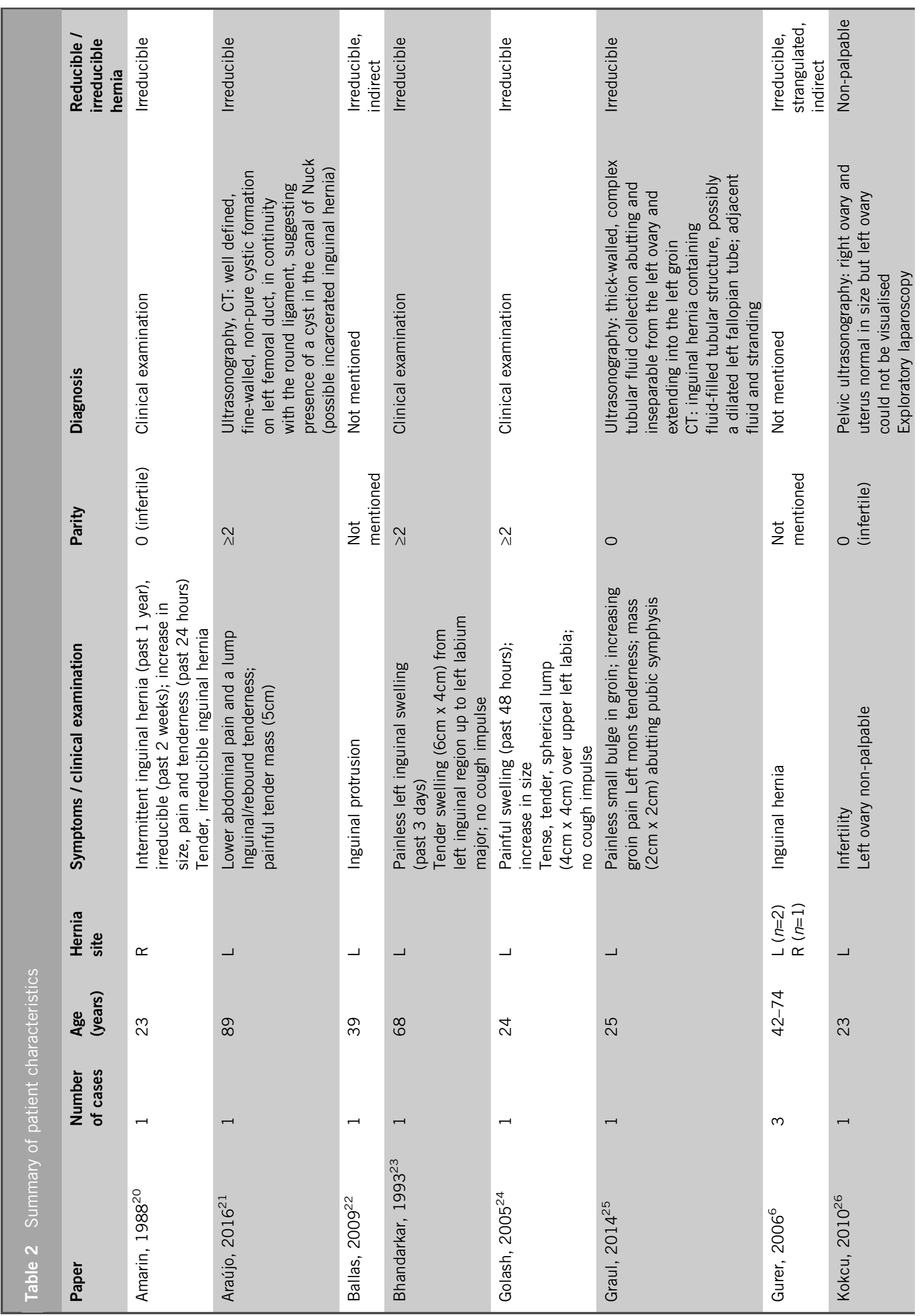



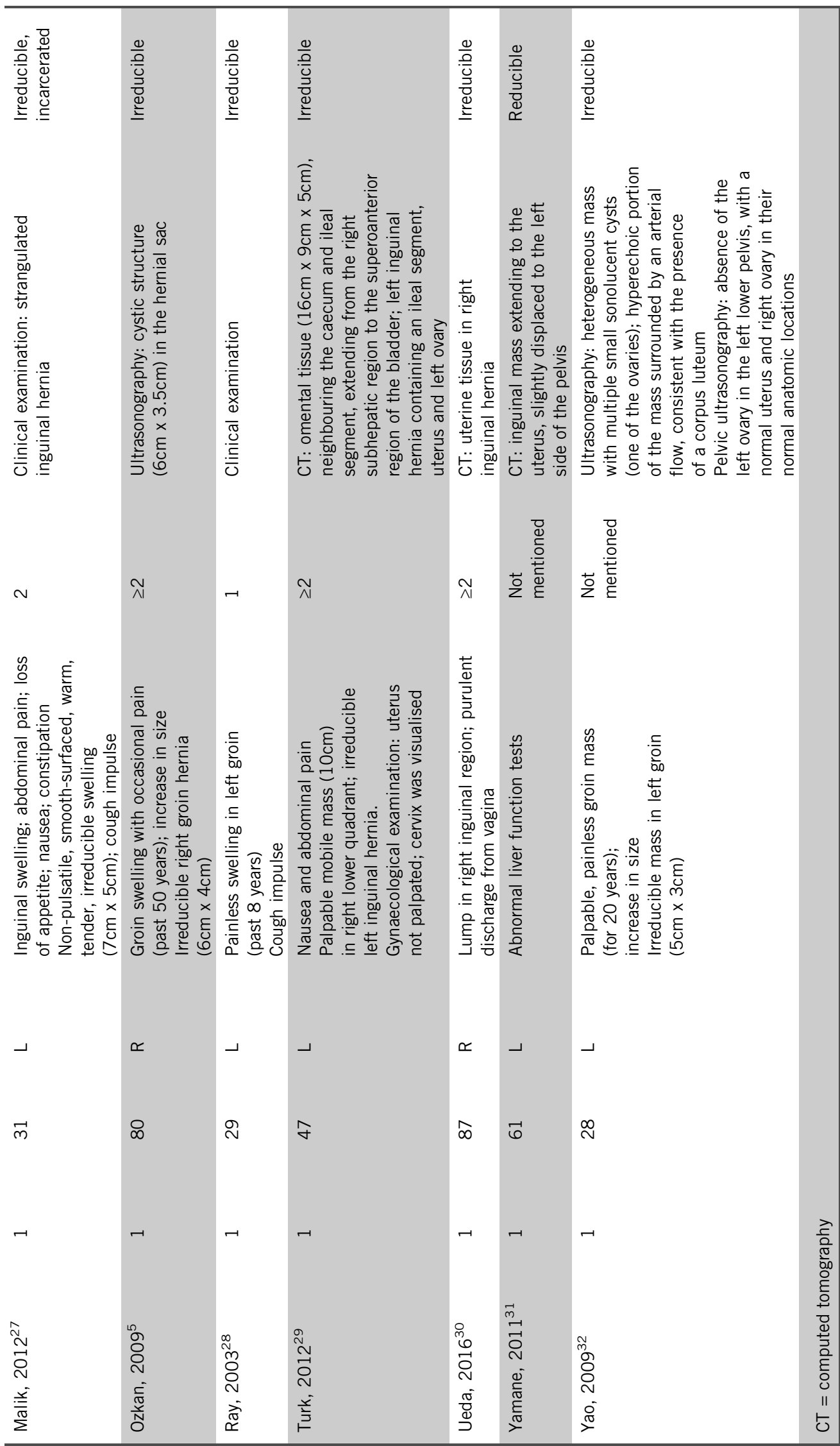


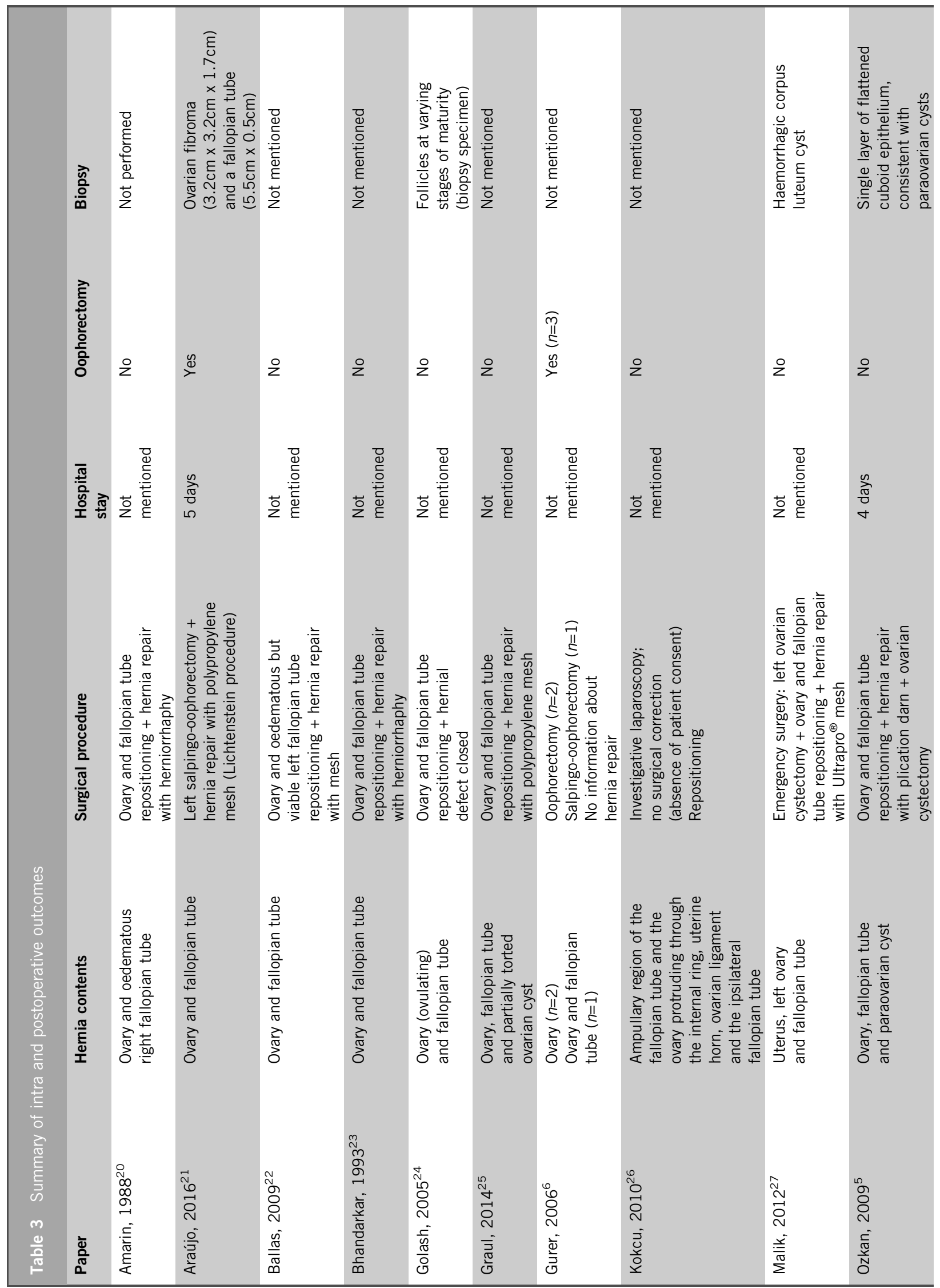


performed surgical procedures. ${ }^{33}$ Occasionally, uncommon intra-abdominal structures may be encountered in a hernial sac. Among the rare contents that can be found in an inguinal hernial sac, gynaecological structures such as the ovaries, fallopian tubes, ligaments and uterus have been described. These findings are common in female infants and young girls, accounting for $6-7 \%$ of inguinal hernias in this subgroup of patients, and are associated with abnormal genital tract development. ${ }^{7}$ Whereas an indirect (and in most cases incarcerated and irreducible) hernia containing an ovary may not be unusual in young girls, it is not commonly reported in women of reproductive age.

There have been case reports of adults presenting with inguinal hernias containing gynaecological structures that were associated with disorders of genital tract development such as Müllerian aplasia or dysplasia and primary amenorrhoea. ${ }^{8,10}$ In these cases, the uterus was the main content found in the hernial sac in women with a $46, \mathrm{XX}$ karyotype who presented with primary amenorrhoea. This was attributed to failure of the Müllerian duct system of the affected site to fuse with the other site and slide into the inguinal canal. As such defects are congenital, we excluded patients with hernias associated with genital tract defects and focused on cases in which a simple prolapse of a gynaecological structure occurred. Given that the reported prevalence of inguinal hernias in women is only $3 \%,{ }^{33}$ this indicates how rare ovarian inguinal hernias are in women with no known developmental genital tract defects.

For the purposes of the present review, a meticulous search of the literature was performed, which yielded a total of 17 cases of ovarian inguinal hernias reported in 15 papers. Nearly half of the patients $(n=8)$ underwent preoperative investigation with CT, ultrasonography or both. Imaging was diagnostic of ovarian content sliding through the inguinal canal in two-thirds of these cases $(n=5,63 \%)$. The diagnosis for the remaining 12 ovarian inguinal hernias was made intraoperatively. Sonographic imaging of the affected area combined with intra-abdominal or transvaginal ultrasonography revealing the absence of one of the two ovaries from the pelvis should enhance the diagnosis of an inguinal hernia. For this reason, the authors of two of the papers included in this review based their preoperative diagnosis on combined ultrasonography of the affected area and pelvic imaging. ${ }^{25,32}$

Of the 17 patients included in our review, 9 (53\%) were women of reproductive age (18-45 years). This finding is remarkable as the sliding of a gynaecological structure through the inguinal canal could theoretically be the result of relaxation of the pelvic ligaments, which is more commonly encountered in postmenopausal women. Ovarian inguinal hernia should be included among the differential diagnoses for women of reproductive age with a groin mass. In this subgroup of women, critical attention should be paid in the preoperative and early diagnosis of a groin mass to avoid delay in diagnosis and consequently torsion or damage of the ovary. In seven of the patients in this review, the ovaries were successfully 
repositioned with the intention to preserve or restore fertility.

Given that $88 \%$ of the patients presented with a groin mass or swelling in the inguinal region, the viability of the gynaecological structures and the difficulty of repositioning them in the peritoneal cavity should be considered before restoration. The authors of five of the papers included in our review reported easy reduction of the contents of the hernial sac into the peritoneal cavity after ensuring that there were no signs of ischaemia. ${ }^{22,24,25,28,30}$

In the case report by Araújo et al, who described an individual who underwent a salpingo-oophorectomy, the reason for excision was not clarified but it is possible that this was due to the advanced age of the patient (89 years). ${ }^{21}$ Gurer et al performed an oophorectomy with and without salpingectomy in two of their patients owing to strangulation. ${ }^{6}$

Three of the patients in our review were nulliparous and had no diagnosed genital defects, and two of these presented with infertility. None of the papers presented outcomes concerning the potential of the women to conceive following hernia repair. This precluded evaluation of the efficacy of early diagnosis and treatment of ovarian inguinal hernia on fertility preservation/restoration. The lack of information regarding the interval between admission and surgery was another limitation of our study, and further discussion is required on the appropriate time to surgery. Despite their rare nature, clinicians should be aware of ovarian inguinal hernias, and should perform clinical and ultrasonography examination of both inguinal regions in these cases, paying special attention to women of reproductive age with a groin lump or in the context of infertility investigation.

To our knowledge, this is the first literature review that presents a cumulative report of adult female patients with inguinal hernias with simple prolapse of gynaecological organs. A thorough search of the literature (with no date or language restrictions) eliminated the risk of missing relevant articles. Nevertheless, the true prevalence of ovarian inguinal hernias could not be ascertained as data concerning their pathophysiology, clinical appearance and treatment are limited to individual case reports and small case series. Furthermore, the significant heterogeneity of the papers included in our review (and the fact that some parameters were omitted by some authors) was another limitation as this means that any conclusions reached must be treated with caution.

\section{Conclusions}

Given the findings of the present literature review, ovarian inguinal hernias should be considered among the differential diagnoses of a groin mass or swelling. The exact mechanisms by which an ovary slides through the inguinal canal remain unclear. Further investigation with imaging should be performed in suspected cases of inguinal masses so that strangulation can be prevented.
Repair of the hernia with the intent to preserve viability of the ovary in women of reproductive age is considered of critical importance.

\section{References}

1. Fitzgibbons RJ, Giobbie-Hurder A, Gibbs JO et al. Watchful waiting vs repair of inguinal hernia in minimally symptomatic men: a randomized clinical trial. JAMA 2006; 295: 285-292.

2. Kingsnorth A, LeBlanc K. Hernias: inguinal and incisional. Lancet 2003; 362 $1,561-1,571$.

3. Pérez Lara FJ, Del Rey Moreno A, Oliva Muñoz H. Do we really know the symptoms of inguinal hernia? Hernia 2015; 19: 703-712.

4. Ramanan B, Maloley BJ, Fitzgibbons RJ. Inguinal hernia: follow or repair? Adv Surg 2014; 48: 1-11.

5. Ozkan OV, Semerci E, Aslan E et al. A right sliding indirect inguinal hernia containing paraovarian cyst, fallopian tube, and ovary: a case report. Arch Gynecol Obstet 2009; 279: 897-899.

6. Gurer A, Ozdogan M, Ozlem $\mathrm{N}$ et al. Uncommon content in groin hernia sac. Hernia 2006; 10: 152-155.

7. Lee J, Scampoli N. Incarcerated ovarian inguinal hernia in a 10-month-old girl. CMAJ 2015; 187: 596-598.

8. Al Omari W, Hashimi H, Al Bassam MK. Inguinal uterus, fallopian tube, and ovary associated with adult Mayer-Rokitansky-Küster-Hauser syndrome. Fertil Steril 2011; 95: 1119.e1-4.

9. Bradshaw KD, Carr BR. Ovarian and tubal inguinal hernia. Obstet Gynecol 1986; 68(3 Suppl): 50S-52S.

10. Elliott DC, Beam TE, Denapoli TS. Hernia uterus inguinale associated with unicornuate uterus. Arch Surg 1989; 124: 872-873.

11. Josefsson ML, Mitra S, Gupta S. Inguinal ovary in adult women - case report and literature review. Springerplus 2013; 2: 545.

12. Kriplani A, Banerjee N, Aminni AC et al. Hernia uterus inguinale in a $46, X X$ female. A case report. J Reprod Med 2000; 45: 48-50.

13. Jategaonkar PA, Yadav SP. Ruptured hemorrhagic ovarian cyst presenting as an incarcerated inguinal hernia in an adult female: a rare clinical scenario of a common surgical emergency. Case Rep Emerg Med 2013; 925694.

14. Machado NO, Machado LS, Al Ghafri W. Laparoscopic excision of a large ovarian cyst herniating into the inguinal canal: a rare presentation. Surg Laparosc Endosc Percutan Tech 2011; 21: e215-e218.

15. Shetty NS, Vallabhaneni S, Patil A et al. Unreported location and presentation for a parasitic ovarian dermoid cyst in an indirect inguinal hernia. Hernia 2013; 17: 263-265

16. Kim JH, Chong GO, Lee JY et al. Laparoscopic repair of indirect inguinal hernia containing endometriosis, ovary, and fallopian tube in adult woman without genital anomalies. Obstet Gynecol Sci 2014; 57: 557-559.

17. Yang $X$, Chen $Q$, Jiang J, Cai X. Irreducible inguinal hernia containing rudimentary uterine horn, ovary, and fallopian tube. Clin Exp Obstet Gynecol 2014; 41: 601-602.

18. Seki A, Maeshima A, Nakagawa $\mathrm{H}$ et al. A subserosal uterus-like mass presenting after a sliding hernia of the ovary and endometriosis: a rare entity with a discussion of the histogenesis. Fertil Steril 2011; 95: 1788.e15-19.

19. Chen CL, Liu TP. Uterus, fallopian tube and ovary within a sliding hernia: a case report. Zhonghua Yi Xue Za Zhi 1994; 53(6 Suppl B): 31-33.

20. Amarin ZO, Hart DM. Inguinal ovary and fallopian tube - an unusual hernia. Int J Gynaecol Obstet 1988; 27: 141-143.

21. Araújo AV, Santos C, Contente H, Branco C. What is inside the hernia sac? BMJ Case Rep 2016; bcr2016215920.

22. Ballas $\mathrm{K}$, Kontoulis $\mathrm{T}$, Skouras $\mathrm{C}$ et al. Unusual findings in inguinal hernia surgery: report of 6 rare cases. Hippokratia 2009; 13: 169-171.

23. Bhandarkar DS, Date RS, Tamhane RG. Irreducible tubo-ovarian inguinal hernia. J Indian Med Assoc 1993; 91: 186.

24. Golash V, Cummins RS. Ovulating ovary in an inguinal hernia. Surgeon 2005; 3: 48 .

25. Graul A, Ko E. Indirect inguinal hernia containing a fallopian tube and ovary in a reproductive aged woman. Case Rep Obstet Gynecol 2014; 437340.

26. Kokcu A, Malazgirt Z, Cetinkaya MB, Tosun M. Presence of a uterine horn and fallopian tube within an indirect hernial sac: report of a rare case. Hernia 2010; 14: 325-327. 
27. Malik KA, Al Shehhi RM, Al Qadhi $\mathrm{H}$ et al. Ovarian hernia: a rarity. Sultan Qaboos Univ Med J 2012; 12: 225-227.

28. Ray S, Som SC, Adedji EA et al. Ovarian and tubal inguinal hernia - an unusual presentation. J Obstet Gynaecol 2003; 23: 323-324.

29. Turk E, Karagulle E, Oguz H, Toprak E. Indirect hernial sac containing the uterus, ovary, and fallopian tube in association with a giant intraabdominal lipoma: report of a case. Hernia 2012; 16: 593-595.

30. Ueda J, Yoshida $\mathrm{H}$, Makino $\mathrm{H}$ et al. Right inguinal hernia encompassing the uterus, right ovary and fallopian tube in an elderly female: case report. J Nippon Med Sch 2016; 83: 93-96.
31. Yamane $\mathrm{H}$, Shiote $\mathrm{Y}$, Harada $\mathrm{D}$, Kamei $\mathrm{H}$. Ovarian inguinal hernia in an elderly female. Intern Med 2011; 50: 2,709.

32. Yao L, Mou Y, Wang HX. Sonographic diagnosis of an ovary-containing inguinal hernia with the formation of a corpus luteum in an adult female. Ultrasound Obstet Gynecol 2009; 34: 359-360.

33. Ge $\mathrm{H}$, Liang $\mathrm{C}, \mathrm{Xu} \mathrm{Y}$ et al. Desarda versus Lichtenstein technique for the treatment of primary inguinal hernia: a systematic review. Int J Surg 2018; 50: 22-27. 